\title{
Desenvolvimento e validação de um roteiro de co-debriefing para o suporte básico de vida simulado
}

\author{
Development and validation of a co-debriefing script for basic life support simulation
}

\author{
Desarrollo y validación de una guia de co-debriefing para el soporte vital básico simulado
}

\begin{abstract}
RESUMO
Objetivo: Desenvolver e validar um roteiro para planejar e executar o co-debriefing na simulação clínica do suporte básico de vida no adulto. Método: Estudo metodológico realizado em duas etapas: revisão de literatura nas fontes National Library of Medicine National Institutes of Health (PubMed ${ }^{\circ}$ ); Scopus; Literatura Latino-Americana e do Caribe em Ciências da Saúde (LILACS) e Cumulative Index to Nursing and Allied Health Literature (CINAHL), utilizando-se o Rayyan para a seleção, e validação por 16 experts, adotando-se o Índice de Validade de Conteúdo. Resultados: Identificaram-se 2694 estudos, e cinco compuseram a amostra, descrevendo: a definição e objetivo do codebriefing; público alvo; objetivos de aprendizagem; recursos materiais; procedimento; observações e referências. Obteve-se um Índice de Validade do roteiro de 0,97. Conclusão: Considerou-se o roteiro válido para planejar e executar o co-debriefing na simulação do suporte básico de vida, contribuindo para enfermagem por sustentar o co-debriefing e potencializar o desenvolvimento de competência clínica.

Descritores: Simulação; Enfermagem; Estudo de Validação; Reanimação Cardiopulmonar; Educação em Enfermagem.
\end{abstract}

\section{ABSTRACT}

Objective: To develop and validate a script to plan and execute the code-debriefing in the clinical simulation of basic life support in adults. Method: Methodological study carried out in two stages: literature review in the sources National Library of Medicine National Institutes of Health (PubMed ${ }^{\circledR}$ ); Scopus; Latin American and Caribbean Health Sciences Literature (LILACS) and Cumulative Index to Nursing and Allied Health Literature (CINAHL), using the Rayyan for selection, and validation by 16 experts, adopting the Validity Index of Contents. Results: 2694 studies were identified, and five composed the sample, describing: the definition and objective of the co-debriefing; target Audience; learning goals; material resources; procedure; observations and references. A script Validity Index of 0.97 was obtained. Conclusion: The script was considered valid to plan and execute the code-debriefing in the simulation of basic life support, contributing to nursing by supporting the code-debriefing and enhancing the development of clinical competence.

Descriptors: Simulation; Nursing; Validation Study; Cardiopulmonary resuscitation; Nursing Education.

\section{RESUMEN}

Objetivo: Desarrollar y validar un guión para planificar y ejecutar el debriefing de código en la simulación clínica de soporte vital básico en adultos. Método: Estudio metodológico realizado en dos etapas: revisión de literatura en fuentes National Library of Medicine National Institutes of Health (PubMed $\left.{ }^{\circledR}\right)$; Scopus; Literatura Latinoamericana y del Caribe en Ciencias de la Salud (LILACS) e Índice Acumulativo de Literatura en Enfermería y Afines en Salud (CINAHL), utilizando el Rayyan para selección y validación por 16 expertos, adoptando el Índice de Validez de Contenidos. Resultados: Se identificaron 2694 estudios y cinco compusieron la muestra, describiendo: definición y objetivo del co-debriefing; Público-objetivo; metas; recursos materiales; procedimiento; observaciones y referencias. Se obtuvo un índice de validez de 0,97. Conclusión: El guión se consideró válido para planificar y ejecutar el code-debriefing en la simulación de soporte vital básico, contribuyendo a la enfermería apoyando el codedebriefing y potenciando la competencia clínica.

Descriptores: Simulación; Enfermería; Estudio de Validación; Reanimación Cardiopulmonar; Educación en Enfermería.
Juliana Silva Garcia Nascimento

$\underline{0000-0003-1118-2738}$

Fabiana Cristina Pires ${ }^{2}$

0000-0002-8524-1449

Daniela da Silva Garcia Regino 2

$\underline{0000-0003-0045-7783}$

Kleiton Gonçalves do

Nascimento $^{2}$

0000-0002-2717-6837

Tainá Vilhar Siqueira ${ }^{1}$

$\underline{0000-0002-1161-5620}$

Maria Celia Barcellos Dalri ${ }^{1}$

0000-0002-8173-8642

1 Universidade de São Paulo.

2 Universidade Federal do Triângulo Mineiro.

Autor correspondente: Juliana Silva Garcia Nascimento

E-mail:

mestradounesp28@yahoo.com.br

\section{Como citar este artigo:}

Nascimento JSG, Pires FC, Regino DSG, et al. Desenvolvimento e validação de um roteiro de co-debriefing para o suporte básico de vida simulado. Revista de Enfermagem do CentroOeste Mineiro. 2021;11:e4085. [Access___]; Available in: DOI:

http://doi.org/10.19175/recom.v11i0. $\underline{4085}$ 


\section{INTRODUÇÃO}

A simulação clínica inserida nos currículos de graduação em enfermagem é uma estratégia de ensino e aprendizagem baseada em evidências, capaz de imitar aspectos de uma situação clínica ou um ambiente e potencializar $o$ desenvolvimento de competência clínica, isto é, o aperfeiçoamento de conhecimentos, habilidades e atitudes ${ }^{(1)}$.

Essa ferramenta pedagógica possui três etapas de importante conceituação e compreensão, visto a necessidade de planejá-las e executá-las de maneira eficaz, a saber: preparação, participação e debriefing ${ }^{(2)}$. A etapa de preparação divide-se em pré-simulação e o prébriefing/briefing. A pré-simulação aborda a leitura, estudo e treinamento de habilidades, para os participantes de uma simulação clínica, anterior à realização do cenário de simulação proposto, e o pré-briefing/briefing caracteriza o esclarecimento dos objetivos de aprendizagem, ambiente, equipamentos, papéis desempenhados pelos participantes, cenário de simulação e tempo, de forma imediata a cena ${ }^{(3)}$.

A participação, etapa intermediária da simulação clínica, caracteriza-se pela execução do cenário pretendido e, por fim, o debriefing, considerado o ponto-chave e o coração do processo de ensino e aprendizagem na simulação clínica, aborda um processo de discussão/reflexão sobre a experiência simulada, no intuito de desenvolver as habilidades cognitivas (conhecimento), psicomotoras (habilidades práticas) e afetivas (atitudes) dos participantes ${ }^{(3)}$.

Acenando para a construção do conhecimento, de maneira ativa, afetiva e colaborativa, a simulação tem ocupado lugar de destaque, desde a formação profissional e, ao longo da vida profissional em enfermagem, principalmente, quando se é necessário estabelecer o processo de ensino e aprendizagem de temas complexos, como a ressuscitação cardiopulmonar e o suporte básico de vida (SBV) ${ }^{(4)}$. O papel fundamental do debriefing, neste contexto, foi recentemente enfatizado pela American Heart Association, que recomendou, em suas novas diretrizes para a ressuscitação cardiopulmonar, a adoção do debriefing para profissionais de saúde, enfatizando seu aspecto de suporte emocional e de análise do desempenho da equipe, para a melhoria da educação e da qualidade do atendimento ${ }^{(4-5)}$.
Desta forma, o debriefing vem sendo recomendado, para potencializar o processo de ensino e aprendizagem na ressuscitação cardiopulmonar e no SBV, coexistindo mais de 30 métodos e 10 técnicas de debriefing que podem ser utilizados para esse fim $^{(6)}$.

O debriefing oral orientado por um único instrutor/facilitador é a técnica comumente adotada, para a simulação do SBV, no entanto acredita-se que um debriefing, realizado por mais de um instrutor/facilitador, denominado codebriefing, seja recomendado nesse âmbito por proporcionar benefícios, como a complementação de estilos de facilitação durante o debriefing e abrangência de diversos tipos de aprendizagem; o desenvolvimento de conhecimentos, habilidades e atitudes pela articulação de expertises dos facilitadores, monitoramento eficaz das expectativas, reações e dificuldades dos participantes, apoio entre os facilitadores na resolução de conflitos; aprimoramento do corpo docente, entre outros ${ }^{(7)}$.

O co-debriefing pode potencializar o processo de ensino e aprendizagem de temáticas complexas como o SBV, à medida que associa o conhecimento de mais do que um instrutor/facilitador e garante atenção às necessidades dos participantes em uma simulação clínica e ao cumprimento dos objetivos de aprendizagem para o $\mathrm{SBV}^{(6-7)}$.

Mesmo diante de seus inúmeros benefícios, adotar o co-debriefing na simulação do SBV pode ser dificultado pela ausência de um instrumento ou roteiro, que auxilie o seu planejamento e execução, guie o instrutor/facilitador durante a aplicação e funcione como um suporte, baseado em evidências científicas confiáveis ${ }^{(7)}$. A falta de um roteiro metodologicamente bem elaborado e validado à aplicação dessa técnica de debriefing confere maior variabilidade, na maneira de executá-lo, o que fragiliza o processo, e isso configura, portanto uma lacuna científica, que instiga a necessidade de se explorar o tema ${ }^{(7)}$, baseando-se na seguinte questão: Quais são os conteúdos necessários para desenvolver um roteiro de co-debriefing capaz de viabilizar o processo de ensino e aprendizagem por meio de simulação clínica sobre o suporte básico de vida no adulto? Diante da importância do co-debriefing, da sua contribuição para o ensino do SBV e inexistência de um padrão, para facilitar a sua execução, este estudo objetivou desenvolver e validar um roteiro para planejar e executar o co- 
debriefing na simulação clínica do suporte básico de vida no adulto.

\section{MÉTODO}

Estudo do tipo metodológico, referente ao desenvolvimento e validação de um roteiro para planejar e executar o co-debriefing, na simulação do SBV no adulto, realizado em uma Universidade pública do interior do Estado de São Paulo, entre junho e novembro de 2020.

As etapas percorridas ${ }^{(8)}$, para estabelecer 0 processo de desenvolvimento e validação desse constructo e os principais referenciais teóricometodológicos que sustentaram esse desenvolvimento, foram: (1) Etapa de procedimento teórico ${ }^{(8)}$ - compilação das evidências científicas que embasaram a identificação dos conteúdos necessários para elaborar o roteiro, baseado no PRISMA ${ }^{(9)}$, um checklist de 27 itens e um fluxograma de quatro etapas, que suportam a qualidade de estudos de revisão, associados às diretrizes da ressuscitação cardiopulmonar atualizadas em 2020(5), para fundamentar aspectos do SBV; (2) Etapa empírica - validação de conteúdo do roteiro por experts da enfermagem e (3) Etapa analítica - análise dos resultados de validação, ambas sustentadas, principalmente, por referenciais teóricometodológicos pertinentes ${ }^{(8,10)}$.

Desta forma, a priori, para o cumprimento da primeira etapa - procedimento teórico, realizou-se uma revisão integrativa da literatura, em julho de 2020, cumprindo-se os passos ${ }^{(11)}$ : identificação do tema e questão norteadora; busca e seleção dos estudos na literatura; categorização; análise dos estudos selecionados e apresentação da revisão. A principal pretensão dessa revisão foi identificar os conteúdos necessários para elaborar um roteiro capaz de sustentar o planejamento e execução do codebriefing no SBV no adulto.

Adotou-se, para este propósito, a estratégia Patient-Intervention-Comparison-Outcomes

(PICO), visto que a Prática baseada em Evidências (PBE) propõe que os problemas clínicos, que surgem na prática assistencial, de ensino ou pesquisa, sejam decompostos e organizados, utilizando-a, o que possibilita a construção de questões de pesquisa de naturezas diversas, de forma adequada, maximiza a recuperação de evidências nas bases de dados, com foco no escopo da pesquisa e evita a realização de buscas desnecessárias ${ }^{(12)}$.
Desta maneira, o acrônimo P (população) foi representado, no presente estudo, por estudantes e profissionais de enfermagem, o acrônimo I (intervenção) abrangeu a síntese das evidências sobre os conteúdos necessários para elaborar um roteiro para o co-debriefing e o acrônimo $O$ (desfecho) foi delineado pelo desenvolvimento do processo de ensino e aprendizagem por meio da simulação clínica. Não foi necessária a adoção do acrônimo C, determinado pela comparação de uma intervenção padrão e uma nova intervenção. A pergunta de pesquisa configurou-se em: Quais as evidências científicas disponíveis na literatura sobre a técnica de co-debriefing para o processo de ensino e aprendizagem de estudantes e profissionais de enfermagem por meio da simulação clínica?

Realizou-se a busca nas seguintes fontes de informação: PubMed ${ }^{\circ}$, Scopus, Cumulative Index to Nursing and Allied Health Literature (CINAHL) e Literatura Latino-Americana e do Caribe em Ciências da Saúde (LILACS).

No PubMed ${ }^{\circ}$ e no Scopus, determinaram-se descritores controlados, na língua inglesa, identificados no Medical Subjects Headings (MeSH): "Students, Nursing"; Nurses; "Simulation Training"; Learning $e$ as palavras-chave: Debriefing; "Co-debriefing" e "Co-facilitation". Realizou-se a seguinte estratégia: ((Students, Nursing" [MeSH Terms]) AND (Nurses [MeSH Terms]) AND ("Simulation Training" [MeSH Terms]) AND (Debriefing OR Co-debriefing "[All Fields] OR Co-facilitation "[All Fields]) AND (Learning [MeSH Terms])).

$\mathrm{Na}$ CINAHL, os descritores controlados foram identificados em Títulos, na língua inglesa, espanhol e português: "Students, Nursing"; Nurses; Simulations; Learning e as palavras-chave: Debriefing; "Co-debriefing" e "Co-facilitation". Procedeu-se à estratégia: SU((Students, Nursing" AND Nurses AND Simulations AND Debriefing OR Co-debriefing OR Co-facilitation AND Learning)).

$\mathrm{Na}$ LILACS, os descritores controlados estavam presentes no Descritores de Ciências da Saúde (Decs), em inglês, português e espanhol: "Students, Nursing"; Nurses; "Simulation Training" e Learning e as palavras-chave: Debriefing, Codebriefing e Co-facilitação.

Realizou-se a seguinte estratégia em português: MH((Estudantes de Enfermagem) AND (Enfermeira e Enfermeiro) AND (Treinamento por Simulação) AND (Debriefing OR Co-debriefing OR 
Co-facilitation) AND (Learning)). Em espanhol: MH((Estudiantes de enfermería) AND (Enfermeras $y$ Enfermeros) AND (Entrenamiento Simulado) AND (Debriefing OR Co-debriefing OR Cofacilitación) AND (Aprendizaje)). Em inglês: $\mathrm{MH}(($ Students, Nursing") AND (Nurses) AND (Simulation Training) AND (Debriefing OR Codebriefing OR Co-facilitação) AND (Learning)). Justifica-se que as palavras-chave Debriefing; Codebriefing; Co-facilitation foram adotadas, com a intenção de alinhar a estratégia de busca especificamente para o objeto de estudo pretendido - o co-debriefing.

Incluíram-se estudos primários, que responderam à questão norteadora, sem delimitação de recorte temporal e idioma, publicados em periódicos científicos e disponíveis eletronicamente. Consideraram-se, também, estudos descritivos que abordaram a opinião de especialistas sobre a técnica de co-debriefing, pela incipiência de publicações sobre a temática. Revisões da literatura, estudos de caso, dissertações, teses, monografias e resumos publicados em anais de eventos foram excluídos.

Após realizar a busca, selecionaram-se os artigos, por meio de três etapas: a primeira de seleção abordou a avaliação de títulos e resumos dos estudos identificados, por dois profissionais, experientes no âmbito da simulação clínica em ressuscitação cardiopulmonar no adulto, com o apoio de um programa de revisão gratuito da web de versão única, chamado Rayyan Qatar Computing Research Institute (Rayyan QCRI) ${ }^{(13)}$, encontrado no link: https://rayyan.qcri.org, que proporciona a seleção de estudos de forma organizada e rápida e permite exportar os artigos de uma base de dados para o programa, com o cegamento do pesquisador auxiliar, garantindo a confiabilidade e precisão metodológica do processo $^{(13)}$.

Na segunda etapa de seleção, 17 estudos apontaram divergência entre os pesquisadores, entregues a um terceiro, responsável por tomar a decisão de inclusão ou exclusão. Na terceira etapa de seleção, realizou-se a leitura na íntegra dos estudos selecionados, definindo-se a amostra final. Ressalta-se que pela escassez de manuscritos identificados sobre o tema, realizouse uma análise das referências dos artigos incluídos, no entanto esse procedimento não resultou em novos acréscimos para a amostra final.
Para extrair as informações dos estudos, utilizou-se um instrumento validado ${ }^{(14)}$, considerando para a presente pesquisa os seguintes critérios: identificação do artigo com título, autores, nível de evidência, país de origem, idioma, ano da publicação, objetivos, delineamento metodológico e resultados. Por fim, classificou-se o nível de evidência dos estudos ${ }^{(15)} \mathrm{e}$ a sua seleção foi demonstrada, conforme recomendação Preferred Reporting Items for Systematic Reviews and Meta-Analyses $(\text { PRISMA) })^{(9)}$.

Em seguida, estruturou-se um roteiro, para a simulação do SBV no adulto e procedeu-se à etapa de procedimento empírico ${ }^{(8)}$, em setembro de 2020, para a validação desse constructo. Inicialmente, por meio da Plataforma Lattes, descreveu-se a estratégia para a busca dos juízes no item modo de busca: busca por assunto selecionou-se: simulação em enfermagem; no item bases, especificaram-se: doutores; no item formação acadêmica/titulação: Doutorado; no item atuação profissional, descreveram-se: grande área, Ciências da Saúde e enfermagem como área.

Consideraram-se os seguintes critérios $^{(16)}$ para a escolha dos juízes: 4 pontos para a titulação de Doutor com tese na área de interesse do estudo; 3 pontos para a titulação de Doutor; 3 pontos para a titulação de Mestre com dissertação na área de interesse do estudo; 2 pontos para a titulação de Mestre; 2 pontos para a publicação de artigo em periódico de referência na área de interesse do estudo; 2 pontos para a experiência profissional de, no mínimo, 2 anos na área de interesse. Determinou-se o valor mínimo de 5 pontos para seleção dos juízes para a validação de conteúdo do roteiro ${ }^{(16)}$.

Um total de 29 juízes foram identificados, dentre os quais 20 obtiveram maior pontuação (10 pontos), selecionados para o contato pela pesquisadora, por e-mail, identificados em seus currículos, nas instituições nas quais trabalhavam e em artigos publicados. Para a compreensão da proposta de validação, enviou-se uma descrição da pesquisa, os objetivos pretendidos e o Termo de Consentimento Livre e Esclarecido (TCLE) para os juízes selecionados. Dos 20 juízes que obtiveram maior pontuação curricular, enquadrando-se nos critérios estabelecidos ${ }^{(16)}, 0$ total de 16 juízes aceitaram participar do processo de validação, para os quais foi enviado um instrumento, em outubro de 2020, baseado em 
uma escala Likert e elaborado por meio do de uma ferramenta eletrônica gratuita denominada Google Forms.

$O$ instrumento para a coleta foi composto por três partes: (A) caracterização dos juízes; (B) conteúdo do roteiro, (C) critérios gerais de avaliação de conteúdo ${ }^{(8)}$, que abordou: critério comportamental (o instrumento é aplicável, com instruções claras e exequíveis); objetividade (as recomendações permitem que se alcance 0 objetivo desejado); simplicidade (os itens expressam uma única ideia e permitem compreensão adequada); clareza (o conteúdo é explicitado de forma clara e inequívoca); relevância (o instrumento é relevante e atende à finalidade proposta); precisão (cada item do instrumento é distinto dos demais; eles não se confundem); variedade (a linguagem é adequada e permite interatividade do conteúdo); modalidade (o vocabulário é adequado, sem gerar ambiguidades); tipicidade (o vocabulário é condizente com a temática, com conceitos adequados); credibilidade (a formulação do instrumento contribui com uma atitude favorável de utilização e compreensão do conteúdo); amplitude (o conteúdo é atual e consistente, com profundidade suficiente para a compreensão do tema); equilíbrio (a sequência proposta apresenta-se de forma equilibrada e coerente).

As opções para a avaliação configuravam-se em: concordo fortemente (4), concordo (3), não sei (0), discordo (2) e discordo fortemente (1), com espaço aberto para "comentários e sugestões". Os juízes tiveram 30 dias para retornarem suas avaliações.

Cumpriu-se, por fim, a etapa de procedimentos analíticos ${ }^{(8)}$, em novembro de 2020, organizando-se, primeiramente, os achados referentes à validação dos juízes, em uma planilha no programa Microsoft Excel 2010, com dupla digitação por dois pesquisadores. A análise quanto à caracterização dos juízes deu-se por meio de estatística descritiva, frequência, percentagem e média, realizada com o apoio do programa Statistical PacKage for the Social Sciences (SPSS), versão 22 para Windows.

Para a avaliação de concordância interavaliadores, considerou-se uma escala do tipo Likert, com pontuação de 1 a 4 para caracterizar a relevância/representatividade das respostas dos juízes, caracterizadas por: 1 - item não relevante ou não representativo, equivalente a discordo fortemente; 2- item necessita de grande revisão para ser representativo, equivalente a discordo; 3 - item necessita de pequena revisão para ser representativo, equivalente a concordo e 4- item relevante ou representativo, equivalente a concordo fortemente ${ }^{(17)}$. A resposta "não sei" não foi pontuada, visto que o juiz não possuía uma opinião concreta e objetiva sobre determinado critério (não concordava e não discordava), e os itens pontuados como 1 ou 2 foram revisados.

Foram utilizados como medida o Índice de Validade de Conteúdo por item (IVC) e o Índice de Validade de Conteúdo total do roteiro ${ }^{(10)}$. Para o cálculo do IVC por item, as respostas foram somadas e avaliadas pelos juízes como valor 3 ou 4 e realizou-se a seguinte fórmula: IVC por item= número de respostas 3 ou 4/número total de respostas/juízes.

Já, para se obter o IVC total do roteiro, utilizou-se o cálculo ${ }^{(10)}$ : IVC total: somatória dos IVCs/número total de itens que compõem o roteiro, isto é: somou-se o valor de IVC de item do roteiro e dividiu-se o resultado pelo número total de itens que o compuseram.

O valor do IVC total do roteiro foi interpretado da seguinte forma ${ }^{(17)}$ : resultado $<0,00$ - pobre concordância; de 0,00 a 0,20- leve concordância; de 0,21 a $0,40-$ aceitável concordância; de 0,41 a 0,60 - moderada concordância; de 0,61 a 0,80- considerável concordância e de 0,81 a 1,00- quase perfeita concordância. Definiu-se, para a presente pesquisa, um IVC total igual ou maior que 0,80 para indicar o conteúdo desse constructo válido ${ }^{(17)}$.

Realizaram-se duas rodadas da técnica Delphi, caracterizada pela análise de um instrumento pelo grupo respondente e de sua concordância ${ }^{(18)}$ Apesar de a primeira rodada ter obtido um IVC total do roteiro acima do estabelecido, para considerar seu conteúdo válido, a segunda rodada priorizou a devolutiva necessária das sugestões realizadas pelos juízes. A pesquisa foi conduzida, de acordo com os padrões éticos exigidos pelas resoluções 466/2012, 510/2016 e 580/2018, do Ministério da Saúde e apresenta o número do protocolo de aprovação 3.826.306 de 6 de fevereiro de 2020 .

\section{RESULTADOS E DISCUSSÃO}

A priori, demonstrou-se a seleção dos estudos científicos incluídos na amostra da 
6| Nascimento JSG, Pires FC, Regino DSG, et al.

presente pesquisa, como se visualiza na Figura 1, a seguir.

Figura 1 - Fluxograma do processo de identificação, seleção e inclusão dos estudos, elaborado a partir da recomendação Preferred Reporting Items for Systematic Reviews and Meta-Analyses (PRISMA)( ${ }^{(9)}$. Ribeirão Preto (SP), Brasil, 2020

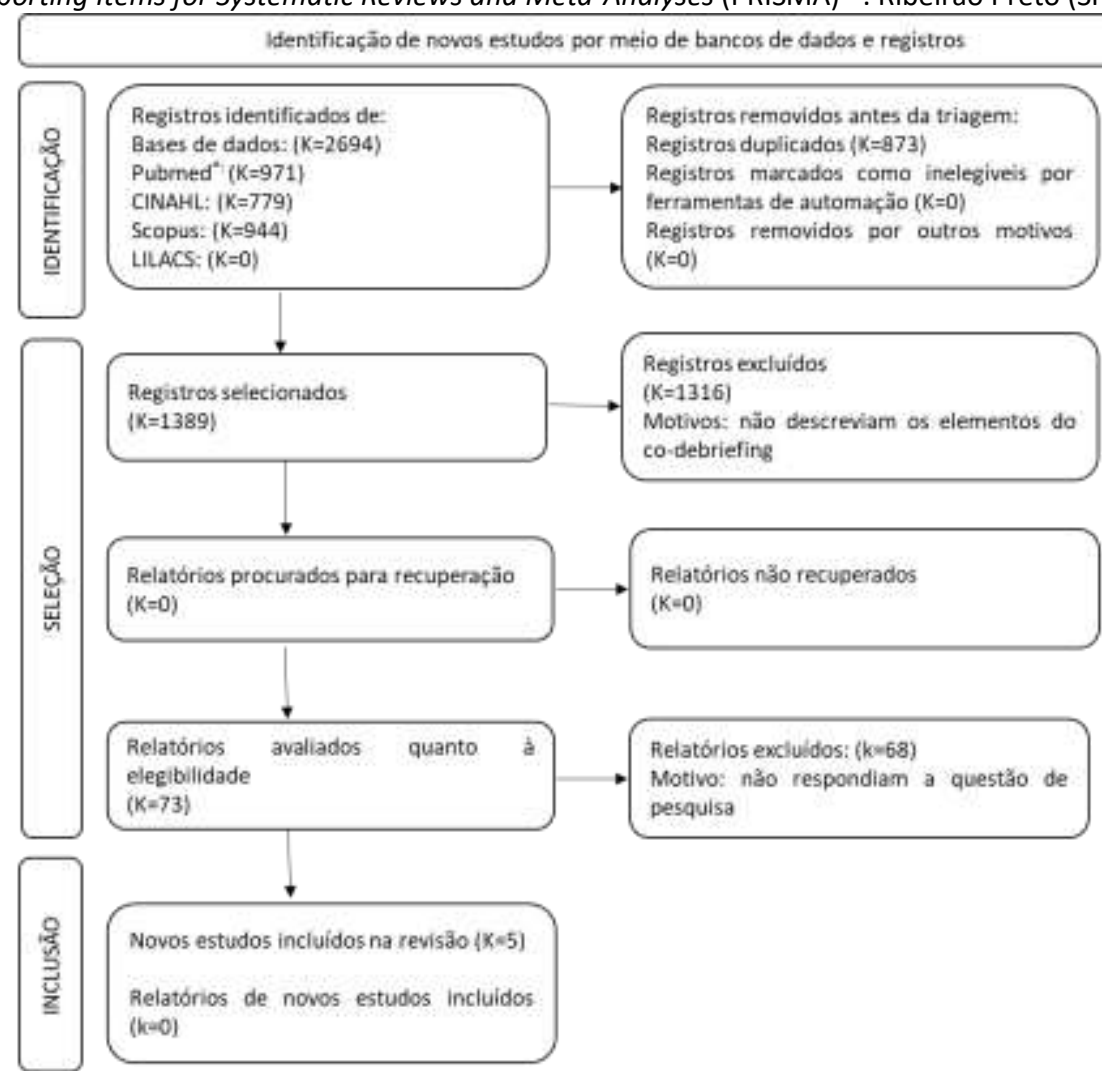

*CINAHL: Cumulative Index to Nursing and Allied Health Literature; LILACS: Literatura Latino-Americana e do Caribe em Ciências da Saúde.

Fonte: dados da pesquisa (2020).

Os estudos científicos sintetizados, por meio da revisão integrativa de literatura, compuseram uma amostra de cinco $\operatorname{artigos}^{(7,19-22)}$ que datam, a partir de 2015, ano de publicação dos primeiros estudos que exploraram o codebriefing e seus conteúdos ${ }^{(7,22)}$; seguido pelo anos de $2016^{(20)} ; 2018^{(19)}$ e $2019^{(2)}$.

A maioria dos manuscritos selecionados apresentaram nível de evidência $6^{(15)}$, caracterizados por estudos descritivos sobre o co- debriefing, baseados na expertise de especialistas $^{(7,20-22)}$. Apenas um estudo quaseexperimental(19) $^{(19 m p a r o u}$ a eficácia do codebriefing com outros tipos de técnicas de debriefing.

Foi possível identificar cinco principais conteúdos pertinentes para o planejamento e execução do co-debriefing, evidenciados na Quadro 1.

Quadro 1 - Conteúdos identificados, por meio de revisão integrativa de literatura, necessários para o planejamento e execução do co-debriefing. Ribeirão Preto, 2020

\section{Conteúdos Descrição dos conteúdos}

\begin{tabular}{|l|l|}
\hline Definição & $\begin{array}{l}\text { Debriefing realizado por mais de um facilitador da mesma ou de diferentes origens profissionais ou } \\
\text { especialidades, para rever um evento real ou simulado, em que os participantes analisam suas ações para } \\
\text { melhorar ou manter o desempenho no futuro(7) }\end{array}$ \\
\hline & $\begin{array}{l}\text { Debriefing realizado por mais de um facilitador da mesma ou de diferentes origens profissionais ou } \\
\text { especialidades, para rever um evento real ou simulado, em que os participantes analisam suas ações para } \\
\text { melhorar ou manter o desempenho no futuro(7) }\end{array}$ \\
\hline Definição
\end{tabular}


Quadro 1 - Conteúdos identificados, por meio de revisão integrativa de literatura, necessários para o planejamento e execução do co-debriefing. Ribeirão Preto, 2020

\begin{tabular}{|c|c|}
\hline Conteúdos & Descrição dos conteúdos \\
\hline Objetivos & $\begin{array}{l}\text { Proporcionar que os facilitadores trabalhem juntos e em colaboração para gerenciar a discussão de forma } \\
\text { fluida(21) } \\
\text { Promover aprendizagem mais eficaz pela união de diferentes perspectivas profissionais(19,22) } \\
\text { Maximizar a qualidade do debriefing, integrando um educador especialista em simulação com um especialista } \\
\text { em conteúdo(7,20-21) }\end{array}$ \\
\hline $\begin{array}{l}\text { Tipos de } \\
\text { debriefing }\end{array}$ & $\begin{array}{l}\text { Abordagem "seguir o líder": identifica um facilitador como o líder, que é responsável por guiar a discussão, } \\
\text { priorizar tópicos e gerenciar o tempo para cada tópico(7) } \\
\text { O facilitador "associado" pode ajudar o facilitador principal a manter o foco, controlar o tempo, ou preencher } \\
\text { lacunas(21) } \\
\text { A abordagem "dividir e conquistar": descreve um processo em que os facilitadores decidem, antes da } \\
\text { simulação ou debriefing, quais tópicos eles abordarão, a ordem em a abordagem ocorrerá e quem liderará a } \\
\text { discussão de cada tópico(7) } \\
\text { A abordagem "pingue-pongue": facilitador e cofacilitador dividem o debriefing, realizando os } \\
\text { questionamentos e reflexões, um após o outro, independentemente de sua expertise }\end{array}$ \\
\hline Estrutura & $\begin{array}{l}\text { (1) Pré-debriefing (antes que o debriefing ocorra); (2) debriefing; e (3) pós-debriefing (após o debriefing } \\
\text { ocorrer)(7) }\end{array}$ \\
\hline Procedimento & $\begin{array}{l}\text { Pré-debriefing: antes que o debriefing ocorra, estratégias podem ser usadas para evitar problemas no } \\
\text { codebriefing: } \\
\text { - Os facilitadores devem se reunir para se familiarizarem com os objetivos de aprendizagem(7,19) } \\
\text { - Estabelecer as "regras de engajamento", incluindo o esclarecimento dos papéis dos facilitadores e } \\
\text { responsabilidades, como, por exemplo, como lidar com interrupções e transições, determinar limites de } \\
\text { tempo para o cenário e debriefing e discutir quais métodos de debriefing e técnicas serão utilizados(7,21) } \\
\text { - Rever brevemente áreas de especialização e discutir como sua expertise será aplicada durante a sessão(7) } \\
\text { Durante o debriefing: } \\
\text { - Estabelecer e manter um ambiente de aprendizado participativo } \\
\text { - Manter "confidencialidade" em relação ao desempenho dos participantes durante debriefing(7,20-22) } \\
\text { - Posicionar-se de frente um para o outro, durante o debriefing, para serem capazes de observar } \\
\text { coletivamente a linguagem corporal e expressões faciais de todos os estudantes e fazer contato visual } \\
\text { claro(7,19-22) } \\
\text { - Promover a comunicação transparente entre facilitadores(21) } \\
\text { - "Checagem de pulso" é a estratégia usada por um facilitador quando está preocupado que a mensagem } \\
\text { passada pelo seu cofacilitador não é clara(7,19-20) } \\
\text { - Evitar a mudança de objetivos e a interrupção da corrente de pensamento ou comentário do outro } \\
\text { facilitador(7) } \\
\text { - Evitar o estilo de "palestra" ou falar de forma direcionada a participantes de uma profissão específica(7,19-20) } \\
\text { - Solicitar verbalmente permissão para interromper(7) } \\
\text { - Perguntar e ouvir o ponto de vista do outro instrutor (21-22) } \\
\text { - Evitar culpar participantes por suas ações ou usar tom de voz crítico ou acusatório(7) } \\
\text { Após o debriefing } \\
\text { - Reunir-se brevemente para discutir questões que surgiram durante o debriefing, para evitar } \\
\text { desentendimento futuro e englobar as regras de engajamento para codebriefing } \\
\text { (7,19-22) }\end{array}$ \\
\hline
\end{tabular}

Fonte: dados da pesquisa, 2020

Quanto à validação de conteúdo do roteiro, dos 16 (100\%) juízes enfermeiros, a maioria era do sexo feminino $(68,8 \%)$, com idade média de 39 anos e tempo de experiência profissional em enfermagem de, em média, 17 anos. A maioria (14-87,5\%) era doutores e docentes no Ensino Superior, e um total de 15 juízes $(93,8 \%)$ possuíam treinamento em simulação, artigos publicados nesta temática e participação em eventos sobre simulação.
Todos os juízes (16-100,0\%) planejaram e desenvolveram simulações clínicas como estratégia de ensino e aprendizagem em enfermagem e dominavam a temática da ressuscitação cardiopulmonar com SBV. A concordância interavaliadores considerou os conteúdos que compuseram o roteiro e os 12 critérios para validação de conteúdo ${ }^{(8)}$, como apresentado na Tabela 1. 
Tabela 1 - Distribuição das respostas dos juízes (16), Índice de Validade de Conteúdo por item e Índice de Validade de Conteúdo total do roteiro sobre o co-debriefing na simulação clínica no suporte básico de vida. Ribeirão Preto, SP, 2020

\begin{tabular}{|c|c|c|c|c|c|c|c|}
\hline \multirow[t]{2}{*}{ Itens para avaliação } & & vância d & esposta & 0 a 4) & & \multirow[t]{2}{*}{$\begin{array}{c}\text { Respostas } \\
\text { válidas } \\
\text { (3 e 4) } \\
\text { N(\%) }\end{array}$} & \multirow[t]{2}{*}{$\begin{array}{l}{ }^{*} \text { IVC } \\
(\%)\end{array}$} \\
\hline & $0 \mathrm{~N}(\%)$ & $1 \mathrm{~N}(\%)$ & $2 \mathrm{~N}(\%)$ & $3 N(\%)$ & $4 \mathrm{~N}(\%)$ & & \\
\hline \multicolumn{8}{|l|}{ Conteúdos } \\
\hline Título & & & $1(6,2)$ & $2(12,5)$ & $13(81,2)$ & $15(93,7)$ & 0,93 \\
\hline Definição & & & & $2(12,5)$ & $14(87,5)$ & $16(100)$ & 1,00 \\
\hline Objetivo & & & $1(6,2)$ & $3(18,75)$ & $12(75)$ & $15(93,7)$ & 0,93 \\
\hline Método de co-debriefing & & & & $4(25)$ & $12(75)$ & $16(100)$ & 1,00 \\
\hline Recursos materiais & & & & $5(31,2)$ & $11(68,7)$ & $16(100)$ & 1,00 \\
\hline Procedimento & & & $1(6,2)$ & $6(37,5)$ & $9(56,2)$ & $15(93,7)$ & 0,93 \\
\hline Tempo & $1(6,2)$ & & $1(6,2)$ & $4(25)$ & $10(62,5)$ & $14(87,5)$ & 0,87 \\
\hline Referências & & & $1(6,2)$ & $1(6,2)$ & $14(87,5)$ & $15(93,7)$ & 0,93 \\
\hline \multicolumn{8}{|l|}{ Critérios para validação } \\
\hline $\begin{array}{l}\text { O roteiro é aplicável, com instruções } \\
\text { claras }\end{array}$ & & & & $4(25)$ & $12(75)$ & $16(100)$ & 1,00 \\
\hline $\begin{array}{l}\text { O roteiro permite que se alcance } 0 \\
\text { objetivo }\end{array}$ & & & & $4(25)$ & $12(75)$ & $16(100)$ & 1,00 \\
\hline Os itens expressam uma única ideia & & & & $4(25)$ & $12(75)$ & $16(100)$ & 1,00 \\
\hline $\begin{array}{l}\text { O conteúdo é explicitado de forma } \\
\text { clara }\end{array}$ & & & & $4(25)$ & $12(75)$ & $16(100)$ & 1,00 \\
\hline $\begin{array}{l}O \text { roteiro é relevante e atende à } \\
\text { finalidade }\end{array}$ & & & & $3(18,75)$ & $13(81,2)$ & $16(100)$ & 1,00 \\
\hline $\begin{array}{l}\text { Cada item do roteiro é distinto dos } \\
\text { demais }\end{array}$ & $1(6,2)$ & & & $4(25)$ & $11(68,7)$ & $15(93,7)$ & 0,93 \\
\hline A linguagem é adequada & $1(6,2)$ & & & $4(25)$ & $11(68,7)$ & $15(93,7)$ & 0,93 \\
\hline O vocabulário é adequado & & & $1(6,2)$ & $4(25)$ & $11(68,7)$ & $15(93,7)$ & 0,93 \\
\hline $\begin{array}{l}\text { O vocabulário é condizente com a } \\
\text { temática }\end{array}$ & & & & $5(31,2)$ & $11(68,7)$ & $16(100)$ & 1,00 \\
\hline $\begin{array}{l}\text { A formulação contribui à } \\
\text { compreensão }\end{array}$ & & & & $3(18,75)$ & $13(81,2)$ & $16(100)$ & 1,00 \\
\hline O conteúdo é atual e consistente & & & & $5(31,2)$ & $11(68,7)$ & $16(100)$ & 1,00 \\
\hline A sequência é equilibrada e coerente & & & & $4(25)$ & $12(75)$ & $16(100)$ & 1,00 \\
\hline *IVC total & & & & & & & 0.97 \\
\hline
\end{tabular}

Fonte: dados da pesquisa (2020).

*IVC: Índice de Validade de Conteúdo

A maioria dos conteúdos que compuseram o roteiro e critérios de avaliação obtiveram um IVC por item igual ou maior que 0,90 , considerada uma concordância quase perfeita. Os itens avaliados como discordo foram revisados.

O IVC total do roteiro apresentou valor de 0,97 - quase perfeita concordância, considerado, portanto, como um constructo válido em seu conteúdo, para subsidiar o planejamento e a execução do co-debriefing, na simulação clínica do SBV no adulto.

Em geral, as sugestões e os apontamentos realizados pelos juízes foram analisados, visando ao aperfeiçoamento do instrumento, principalmente, quanto: à adequação do título, tempo e procedimentos. A versão final do roteiro validado (Anexo $A$ ) constituiu-se de sete itens, a saber: (1) definição e objetivo: definiu-se no roteiro o co-debriefing e seus objetivos; (2) público-alvo: definiu-se o público-alvo em que o roteiro poderá ser aplicado; (3) objetivos de aprendizagem para o SBV: descreveram-se os objetivos de aprendizagem que são possíveis de serem atingidos por meio da aplicação do codebriefing; (4) recursos materiais/instrumentos necessários: apontaram-se todos os materiais utilizados para estabelecer essa técnica; (5) procedimento: definiu-se minuciosamente um passo a passo, bem estabelecido para a condução do co-debriefing por instrutores/facilitadores; (6) observações: descreveram-se pontos de reflexão e organização importantes para planejar e executar o co-debriefing; (7) referências. $O$ roteiro foi intitulado: Roteiro para o co-debriefing na simulação clínica do suporte básico de vida (ANEXO A). 
Para que o processo de ensino e aprendizagem do suporte básico de vida seja concretizado com eficiência, faz-se necessário adotar-se estratégias que motivem o estudante e o profissional a serem sujeitos ativos do seu

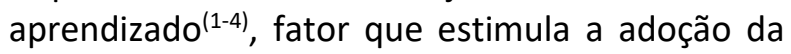
simulação clínica como estratégia pedagógica e do debriefing, como mecanismos que potencializam o desenvolvimento de competência clínica neste âmbito ${ }^{(1)}$.

$\mathrm{O}$ roteiro proposto apresenta como maior potencialidade e benefício a articulação da simulação clínica, como estratégia pedagógica inovadora, para o processo de ensino e aprendizagem em enfermagem, com a temática do suporte básico de vida no adulto e, ainda, confere ineditismo a ciência em enfermagem por estabelecer um padrão baseado em evidências científicas fidedignas, para a condução do codebriefing no SBV, de maneira que possa ser adaptado, também, para outros temas e outras realidades em saúde, o que torna esse instrumento versátil, útil e de fácil manipulação.

Notou-se que a maioria dos artigos que compuseram a amostra selecionada apresentaram baixo nível de evidência, caracterizados por estudos descritivos, que valorizam a opinião de experts na área, e isso se justifica, possivelmente, pela atualidade da temática, visto que os primeiros estudos deram-se em $2015^{(7,22)}$ e propuseram, primeiramente, a sustentação de um referencial teórico pertinente ao co-debriefing, para a estruturação de um corpo de conhecimento capaz de embasar futuros estudos observacionais e experimentais ${ }^{(7,22)}$.

Mesmo que os conteúdos que compuseram o roteiro tenham sido extraídos, na maioria, de manuscritos de nível de evidência 6, do tipo descritivos, foram considerados consistentes e suficientes para desenvolver esse constructo, no entanto enfatiza-se, ainda, a importância de elaborarem-se pesquisas, metodologicamente bem delineadas sobre o codebriefing e sua adoção no processo de ensino e aprendizagem do SBV, que sustentem melhor nível de evidencia dos achados sobre a temática.

Destacou-se como um conteúdo necessário ao roteiro a definição do termo co-debriefing, visto a necessidade de esclarecê-lo e distingui-lo da técnica de peer-debriefing. O peer-debriefing caracteriza-se por uma sessão de discussão realizada, após o cenário simulado, conduzida entre pares de alunos ou participantes de uma simulação clínica, sem a instrução de um facilitador e, muitas vezes, essa técnica é denominada como co-debriefing, de forma errônea, o que enfatiza a importância do roteiro proposto tornar evidente a proposta do codebriefing ${ }^{(7,19,22)}$.

Já, o objetivo do ensino e aprendizagem do SBV, por meio do co-debriefing, é obter-se uma aprendizagem efetiva, por meio de um trabalho em conjunto de dois profissionais, com diferentes expertises, que se alinham e se complementam, para fomentar a discussão e conferir qualidade ao debriefing realizado ${ }^{(7)}$.

Identificaram-se três tipos de co-debriefing: seguir o líder; dividir e conquistar e pinguepongue $^{(7)}$. A principal diferença entre eles está na forma pela qual o instrutor/facilitador conduz a sessão de co-debriefing. No co-debriefing do tipo seguir o líder, há um facilitador principal, e o cofacilitador auxilia nas atividades que foram direcionadas a ele. $\mathrm{Na}$ abordagem dividir e conquistar, inexiste o facilitador líder e há divisão total das atividades, que proporcionam a reflexão durante o co-debriefing, aproveitando da expertise de cada facilitador e, na abordagem do tipo pingue-pongue, os facilitadores conduzem a reflexão um após o outro, independentemente de suas expertise sobre a temática' e, desta forma, mais importante que o tipo de co-debriefing que será executado, é o alinhamento entre os facilitadores para a qualidade da discussão e seguimento correto da técnica ${ }^{(7)}$.

Pelo fato do co-debriefing abordar mais que um facilitador, a sua estrutura e o procedimento à execução são considerados fases de que outros tipos de debriefing não se apropriam ${ }^{(7,21-22)}$. O codebriefing enfatiza uma fase de pré-debriefing, não especificada por nenhum outro tipo de técnica de debriefing, que visa alinhar e planejar o debriefing que será executado entre os facilitadores e, após a sua execução, prioriza-se o pós-debriefing, para a reflexão dos próprios facilitadores sobre as potencialidades e fragilidades daquilo que promoveram e melhoria futura(7).

$\mathrm{O}$ índice de Validade de Conteúdo por critério e o Índice de Validade de Conteúdo total do roteiro foram utilizados como medidas para a análise da sua validade e, apesar da validade de conteúdo ser considerada uma avaliação subjetiva, feita com o objetivo de determinar se a escolha dos itens que compõem o instrumento é adequada, caracteriza-se como uma etapa 
importante quando se propõe desenvolver um novo instrumento ${ }^{(8,10,23)}$.

Os estudos de validação sobre simulação clínica, geralmente, envolvem o desenvolvimento de questionário pré e pós-teste ${ }^{(23)}$ ou cenários de simulação ${ }^{(24)}$, sendo que roteiros para a condução do debriefing são ainda pouco explorados ${ }^{(7)}$.

Um estudo de validação de um cenário de simulação clínica, para o manejo da hemorragia pós-parto, realizado em 2016, assemelhou-se aos resultados de validação da presente pesquisa, ao alcançar um IVC total estudo de 0,95, capaz de considerá-lo válido em seu conteúdo para a capacitação dos alunos de enfermagem ${ }^{(24)}$.

Corrobora também com esse mecanismo de validação de conteúdo, pesquisa realizada, em 2019, para validar um checklist de avaliação da capacitação com simulação clínica do atendimento ao paciente séptico, que apresentou um IVC maior que 0,80, apontando o checklist válido em seu conteúdo e útil para a capacitação de profissionais da saúde no atendimento ao paciente séptico, por meio de simulação clínica ${ }^{(25)}$.

Quanto ao ensino do SBV no adulto, viabilizado por meio da simulação clínica e do debriefing, estudo realizado, em 2019, elaborou e validou um questionário sobre o conhecimento do SBV, obtendo-se um constructo válido, composto por 20 questões de múltipla escolha, com concordância interavaliadores "quase perfeita", o que corrobora com a validação de conteúdo apresentada na presente pesquisa ${ }^{(23)}$.

Refletir sobre uma experiência de ensino do SBV, por meio de uma técnica de debriefing, pode parecer natural e esperado, no que diz respeito a um processo pedagógico baseado em simulação clínica, no entanto a ausência de um roteiro que facilite o planejamento e a condução dessa discussão pode resultar em uma forma não sistematizada ou até ineficiente de aprendizagem, o que implica diretamente a necessidade de produzirem-se constructos válidos e fidedignos para esse fim $^{(7,23-25)}$.

\section{CONCLUSÃO}

Os conteúdos utilizados, para se desenvolver um roteiro para planejar e executar o co-debriefing, na simulação clínica do suporte básico de vida no adulto foram a definição e objetivo do co-debriefing; público- alvo; objetivos de aprendizagem para o SBV; recursos materiais/instrumentos necessários; procedimento; observações; referências.
Considerou-se o roteiro válido em seu conteúdo por apresentar um Índice de Validade de Conteúdo total de 0,97 , caracterizado como quase perfeita concordância, que pondera o constructo adequado e pertinente para o processo de ensino e aprendizagem do Suporte Básico de Vida no adulto na simulação clínica.

Esta pesquisa obteve como principais limitações, a priori, a identificação de uma amostra reduzida de estudos que expõe os conteúdos necessários, para planejar e executar o co-debriefing e, principalmente, a necessidade de desenvolverem-se pesquisas de intervenção para alcançar níveis de evidência mais consistentes quanto a essa temática.

Este instrumento contribui com a pesquisa, ensino e assistência, tanto na enfermagem quanto para outras áreas profissionais em saúde, por estabelecer um padrão confiável para condução do co-debriefing, que potencializa o desenvolvimento de competência clínica e confere qualidade e segurança ao processo de ensino e aprendizagem.

\section{REFERÊNCIAS}

1 - Garner SL, Samyappan J, Cyriac R, Vidhya P, Selva FE, Muggalla DS. Simulation evaluation: observation versus self-efficacy among nursing students in India. Clin Simul Nurs. 2020;39:55-61. DOI: $10.1016 /$ i.ecns.2019.11.005

2 - Lubbers J, Rossman C. Satisfaction and selfconfidence with nursing clinical simulation: novice learners, medium-fidelity, and community settings. Nurse Educ Today 2017;48:140-4. DOI: 10.1016/j.nedt.2016.10.010

3 - Tyerman J, Flude ML, Graham L, Coffey S, Lynch EO. A systematic review of health care presimulation preparation and briefing effectiveness. Clin Simul Nurs. 2019;27:12-25. DOI: 10.1016/j.ecns.2018.11.002

4 - Tobase L, Peres HH, Tomazini EA, Teodoro SV, Ramos MB, Polastri TF. Basic life support: evaluation of learning using simulation and immediate feedback devices. Rev Latino-Am Enfermagem 2017;25:e2942. DOI: $1518-$ 8345.1957.2942

5 - American Heart Association (AHA). Destaques das diretrizes de RCP e ACE de 2020 da American Heart Association. Chicago: AHA; 2020 [citado em 
10 out 2020]. Available in: https://international.heart.org/pt

6 - Kang K, Yu M. Comparison of student selfdebriefing versus instructor debriefing in nursing simulation: a quasi-experimental study. Nurse Educ Today 2018;2:67-73. DOI: 10.1016/j.nedt.2018.02.030

7 -Cheng A, Palangas J, Eppich W, Rudolph J, Robinson T, Grant V. Co-debriefing for simulationbased education: a primer for facilitators. Simul Healthc. 2015;10(2):69-75. DOI: $\underline{10.1097 / \mathrm{SIH} .0000000000000077}$

8 - Pasquali L, organizador. Instrumentação psicológica: fundamentos e práticas. Porto Alegre: Artmed; 2010.

9 - Page MJ, McKenzie JE, Bossuyt PM, Boutron I, Hoffmann TC, Mulrow CD, et al. The PRISMA 2020 statement: an updated guideline for reporting systematic reviews. 2020;1-36. DOI: /10.31222/osf.io/v7gm2

10 - Polit DF, Beck CT. The Content Validity Index: are you sure you know what's being reported? Critique and recommendations. Res Nurs Health 2006;29(5):489-97. DOI: 10.1002/nur.20147

11 - Whittemore R, Knafl K. The integrative review: updated methodology. J Adv Nurs. 2005;52(5):54653. DOI: /10.1111/j.1365-2648.2005.03621.x

12 - Santos CM, Pimenta CA, Nobre MR. The pico strategy for the research question construction and evidence search. Rev Latino-Am Enfermagem 2007;15(3):508-11. DOI: 10.1590/S0104$\underline{11692007000300023}$

13 - Ouzzani M, Hammady H, Fedorowicz Z, Elmagarmid A. Rayyan-a web and mobile app for systematic reviews. Syst Rev. 2016;5(1):210. DOI: 10.1186/s13643-016-0384-4

14 - Ursi ES, Galvão CM. Perioperative prevention of skin injury: an integrative literature review. Rev Latino-Am Enfermagem 2006;14(1):124-31. DOI: $\underline{10.1590 / \text { S0104-11692006000100017 }}$

15 - Melnyk BM, Fineout-Overholt E. Evidencebased practice in nursing \& healthcare: a guide to best practice. Philadelphia: Wolters Kluwer Health; 2011.
16 - Fehring RJ. The Fehring model. In: CarrolJohnson RM, Paquete M. Classification of nursing diagnoses: proceeding of the tenth conference. Philadelphia: Lippincott Company; 1994. p. 58-63.

17 - Landis JR, Koch GG. The measurement of observer agreement for categorical data. Int J Biom. 1977;33(1):159-74. DOI: 10.2307/2529310

18 - Balasubramanian R, Agarwal D. The Delphi technique: a review. J Int Soc Prev Community Dent. 2012;16(2):16-25. DOI: 10.12968/ijtr.2009.16.2.38892

19 - Brown DK, Wong AH, Ahmed RA. Evaluation of simulation debriefing methods with interprofessional learning. J Interprof Care 2018;32(6):779-81. DOI: 10.1080/13561820.2018.1500451

20 - Krogh K, Bearman M, Nestel D. "Thinking on you feet": a qualitative study of debriefing practice. Adv Simul. 2016;1(1):1-12. DOI: 10.1186/s41077016-0011-4

21 - Maestre JM, Rojo E, Piedra L, Moral I, Simon R. El experto en contenidos como instructor colaborador en el debriefing. Simulación Clínica 2019 [citado em 1 nov 2020]; 1(1):37-44. Available in:

https://www.medigraphic.com/pdfs/simulacion/rs c-2019/rsc191g.pdf

22 - Greiff JL, Bricker MG, Gamaghelyan P, Tadevosyan $\mathrm{M}$, Deng $\mathrm{S}$. Debriefing in peacemaking and conflict resolution practice: models of emergent learning and practitioner support. Reflective Practice 2015;16(2):254-68. DOI: $10.1080 / 14623943.2015 .100558923$

23 - Alves MG, Pereira VO, Batista DF, Cordeiro AL, Nascimento JS, Dalri MC. Construction and validation of a questionnaire for cardiopulmonary resuscitation knowledge assessment. Cogitare Enferm. 2019;24:e64560. DOI: 10.5380/ce.v24i0.64560

24 - Andrade PO, Oliveira SC, Morais SC, Guedes TG, Melo GP, Linhares FM. Validation of a clinical simulation setting in the management of post partum haemorrhage. Rev Bras Enferm. 2019;72(3):624-31. DOI: 10.1590/0034-71672018-0065

25 - Lino RL, Oliveira SA, Silva KP, Machado RC. Checklist validation for evaluation of training with 
12 | Nascimento JSG, Pires FC, Regino DSG, et al.

clinical Simulation of septic patient care. Enferm

Glob. 2019;18(56):185-97. DOI:

10.6018/eglobal.18.4.341171

Nota: Este estudo é um recorte da tese de doutorado intitulada "Efetividade do co-debriefing na simulação clínica do suporte básico de vida: estudo piloto" para obtenção do título de doutor pelo Programa de Pós-graduação em Enfermagem Fundamental da Escola de Enfermagem de Ribeirão Preto. Financiamento da Coordenação de Aperfeiçoamento de Pessoal de Nível Superior (CAPES).

Received in: 09/11/2020

Approved in: 23/12/2020 
ANEXO A - Roteiro para o co-debriefing na simulação clínica do Suporte Básico de Vida

\begin{tabular}{|c|c|c|}
\hline ELEMENTOS & DESCRIÇÃO & TEMPO \\
\hline Definição e objetivo & $\begin{array}{l}\text { O codebriefing é uma técnica de discussão/reflexão realizada por mais de um } \\
\text { facilitador, que associa as perspectivas e saberes de profissionais diferentes, } \\
\text { idealmente, de um educador e de um profissional de saúde atuante da temática } \\
\text { proposta. Existem três tipos de codebriefing: A abordagem "seguir o líder", em que há } \\
\text { um facilitador principal, que guia a discussão e um facilitador associado, que mantém } \\
\text { o foco, controla o tempo e preenche lacunas. A abordagem "dividir e conquistar", em } \\
\text { que os facilitadores decidem antes do debriefing quais tópicos eles abordarão, a ordem } \\
\text { e quem liderará a discussão e a abordagem "pingue-pongue", em que facilitador e } \\
\text { cofacilitador dividem o debriefing, realizando os questionamentos, alternadamente, } \\
\text { independentemente de sua expertise. Objetiva-se desenvolver competências clínicas } \\
\text { para o Suporte Básico de Vida (SBV) no adulto. }\end{array}$ & $\begin{array}{l}\text { O co-debriefing } \\
\text { deve ter } \\
\text { duração } \\
\text { compatível } \\
\text { com o alcance } \\
\text { dos objetivos } \\
\text { educacionais } \\
\text { pretendidos. }\end{array}$ \\
\hline Público alvo & $\begin{array}{l}\text { Profissionais enfermeiros e estudantes de graduação em enfermagem, de preferência, } \\
\text { que já tenham tido contato com o ambiente hospitalar, durante as práticas } \\
\text { estabelecidas pela universidade e com a disciplina de bases técnicas. }\end{array}$ & \\
\hline $\begin{array}{l}\text { Objetivos de } \\
\text { aprendizagem para } \\
\text { o SBV }\end{array}$ & $\begin{array}{l}\text { Para desenvolver as habilidades cognitivas (conhecimento) e habilidades psicomotoras } \\
\text { (prática): } \\
\text {-Conhecer e compreender a cadeia de sobrevivência intra-hospitalar; } \\
\text {-Analisar a cadeia de sobrevivência intra-hospitalar articulando-a com a experiência } \\
\text { realizada no cenário simulado do SBV; } \\
\text {-Conhecer, compreender e sintetizar a importância da Vigilância e Prevenção, como } \\
\text { primeiro elo da cadeia de sobrevivência intra-hospitalar; } \\
\text {-Analisar e avaliar a importância do reconhecimento imediato da parada } \\
\text { cardiorrespiratória (PCR) e acionamento do Serviço Médico de Emergência; } \\
\text {-Conhecer, compreender, sintetizar, analisar e avaliar as características da } \\
\text { Ressuscitação Cardiopulmonar (RCP) de alta qualidade com SBV: a frequência ideal da } \\
\text { compressão torácica externa (CTE); profundidade da CTE; permissão do retorno do } \\
\text { tórax após a compressão; minimizar as interrupções entre as compressões; } \\
\text { posicionamento ideal das mãos durante a compressão; evitar ventilação excessiva; } \\
\text { relação compressão-ventilação sem via aérea avançada, utilização do Desfibrilador } \\
\text { Externo Automático (DEA). } \\
\text { Para desenvolver as habilidades afetivas (atitudes): } \\
\text {-Ter disposição e atenção para aprender; } \\
\text {-Participar ativamente e com satisfação; } \\
\text {-Estabelecer compromisso com o aprendizado; } \\
\text {-Dar valor a cada situação aprendida e contextualizá-la; } \\
\text {-Transferir o aprendizado experiencial para a prática real. }\end{array}$ & \\
\hline $\begin{array}{l}\text { Recursos } \\
\text { necessários }\end{array}$ & Cadeiras; Folha de sulfite para anotações; Caneta; Prancheta. & \\
\hline Procedimento & $\begin{array}{l}\text { Esse roteiro adota o método G.A.S debriefing (Structured and supported debriefing) } \\
\text { para guiar a reflexão e a técnica de codebriefing "dividir e conquistar", permeada por } \\
\text { três fases distintas: pré-debriefing; debriefing e o pós-debriefing. } \\
\text { O pré-debriefing ocorre antes da realização do cenário proposto, o debriefing, após o } \\
\text { cenário, e a etapa de pós-debriefing, ao término da reflexão, como descrito a seguir: } \\
\text { 1o Pré-debriefing: } \\
\text { - Realizar reunião (cofacilitadores) para se familiarizarem com os objetivos de } \\
\text { aprendizagem; } \\
\text { - Desenvolver uma compreensão compartilhada de como o debriefing será } \\
\text { compartilhado de acordo com as fases do G.A.S debriefing: } \\
\text { Responsável pela etapa "G": } \\
\text { Responsável pela etapa "A": } \\
\text { Responsável pela etapa: "S": } \\
\text { - Estabelecer as regras de engajamento (interrupções e transições): Sinal para transição } \\
\text { de etapa: } \\
\text { Sinal para interrupção do cofacilitador: } \\
\text { Determinando limites de tempo para o cenário e etapas do codebriefing } \\
\text { - Rever brevemente áreas de especialização e discutir como a sua expertise será } \\
\text { aplicada durante a sessão; } \\
\text { - Rever como se desenvolverá a cena do caso de simulação; } \\
\text { - Rever equipamentos, suprimentos, papéis dos atores durante a cena; } \\
\text { - Discutir como gerenciar desentendimentos; } \\
\text { - Determinar quem vai manter o tempo; }\end{array}$ & $\begin{array}{l}\text { O co-debriefing } \\
\text { deve ter } \\
\text { duração } \\
\text { compatível } \\
\text { com o alcance } \\
\text { dos objetivos } \\
\text { educacionais } \\
\text { pretendidos. }\end{array}$ \\
\hline
\end{tabular}


- Posicionamento físico (onde e como sentaremos?);

- Definir comunicação não verbal e linguagem corporal.

\section{$2^{\circ}$ Cenário}

Após realizar a cena, os participantes devem ser conduzidos para um ambiente destinado ao debriefing, dispostos em cadeiras em um semicírculo;

30 Etapa do G.A.S “G” - Gather-

Responsável:

(Etapa de Reunião das informações e sentimentos, também chamada de "Reação");

Objetivo: Ouvir os participantes e entender o que eles pensam e como se sentem sobre a sessão simulada;

Que deve ser feito? Uso de comunicação não verbal: os facilitadores devem posicionarse em frente um ao outro, durante o debriefing, para serem capazes de observar coletivamente a linguagem corporal e expressões faciais de todos dos alunos e cofacilitadores;

Expressar o "princípio básico" para a tranquilidade dos participantes: _ "Acreditamos que todos os participantes são capazes e se importam em fazer bem o seu trabalho e querem melhorar". Solicitar a narrativa da equipe sobre a vivência.

Questões realizadas: Como vocês se sentem? Vocês podem nos dizer o que aconteceu quando...? Que vocês podem adicionar?

Pré-visualização (descreve a verbalização deliberada por um facilitador da intenção de transições de um tópico para o próximo;

40 Etapa: "A" - Analyze

Responsável

(Etapa para análise das informações e articulação com o referencial teórico que embasou a cena, também chamada de "Compreensão")

Objetivo: Facilitar a reflexão e a análise dos participantes quanto a suas ações;

Que deve ser feito? Solicitar instrução da equipe sobre a vivencia, relatório verbal das observações, ações corretas e que podem ser melhoradas. Fazer uma série de perguntas para revelar os processos de pensamento dos participantes e ajudá-los a refletir sobre seu desempenho;

Questões realizadas: Percebi...Me conte mais sobre...Como você se sentiu sobre...O que você estava pensando quando...Eu entendo, no entanto, me fale sobre o aspecto " $X$ " do cenário...

5o Etapa: "S" - Summarize

Responsável:

(Etapa final, que organiza mentalmente a reflexão e articula o aprendizado com a vida real);

Objetivo: Facilitar a identificação e a revisão das lições aprendidas

Que deve ser feito? Os participantes identificam aspectos positivos quanto ao seu comportamento ou da equipe que exige mudança. Resumo de comentários ou declarações.

Questões realizadas: Liste duas ações ou eventos que você sentiu que foram eficazes ou bem-feitas. Descreva duas áreas que você/a equipe acham que precisa melhorar. Que vocês aprenderam aqui, que levarão para a prática?

60 Etapa: Pós-debriefing

Após o debriefing ter ocorrido, os facilitadores devem reunir-se brevemente para discutir questões que surgiram durante o debriefing. A discussão aberta ajudará a evitar qualquer desentendimento futuro que possa ter ocorrido, durante o debriefing e ajuda a englobar as regras de engajamento para codebriefing e evitar que desafios surjam no futuro.

- Questionar se os principais objetivos de aprendizado ainda são os mesmos.

- Priorizar a discussão dos objetivos de aprendizagem;

- Verificar se houve algum problema que exija atenção ou sensibilidade específica;

- Verificar se houve abordagem adequada de todos os objetivos de aprendizagem prédefinidos;

- Verificar tópicos com necessidade de melhoria;

- Questionar se houve bom uso da expertise coletiva;

- Questionar se a abordagem adotada foi coerente;

- Estabelecer pontos fortes e o que é preciso melhorar;

- Questionar se: o método de debriefing foi eficaz, pontos fortes, pontos a serem melhorados, necessidade de interrupções, como ocorreu a transição entre os tópicos de discussão, ocorrência de desentendimentos, como o tempo foi gerenciado, se o posicionamento dos facilitadores foi eficaz, se os métodos de comunicação não verbal foram eficazes; 


\begin{tabular}{|c|c|}
\hline Observações & $\begin{array}{l}\text { - Estabelecer e manter um ambiente de aprendizado participativo; } \\
\text { - Manter "confidencialidade" em relação ao desempenho dos participantes durante } \\
\text { debriefing; } \\
\text { - Ouvir, observar e refletir (ao ouvir ativamente a discussão e observar a linguagem } \\
\text { corporal dos alunos, os facilitadores podem prever melhor a linha antecipada de } \\
\text { questionamento de seu cofacilitador e de forma mais eficaz identificar quando e como } \\
\text { contribuir para a conversa sem interrupções); } \\
\text { - Negociação Aberta (promover a comunicação transparente entre facilitadores); } \\
\text { Evite mudar o objetivo de aprendizagem, antes de terminar o que está sendo discutido, } \\
\text { interromper a corrente de pensamento ou comentário do outro instrutor várias vezes } \\
\text { seguido em um assunto particular (com raras exceções); } \\
\text { - Evite o estilo de conferência, domine ou assuma a discussão, ou fale diretamente ou } \\
\text { apenas com participantes de uma profissão específica; } \\
\text { - Evite ignorar o bom ou excelente desempenho, ou ignorando os principais pontos de } \\
\text { aprendizagem; } \\
\text { - Solicitar verbalmente permissão para interromper: } \\
\text { - Forneça uma breve pré-visualização do tópico sobre o qual você deseja falar (por } \\
\text { exemplo: "Eu gostaria de falar sobre o tópico da conversa"); } \\
\text { - Pergunte e ouça o ponto de vista do outro instrutor: "Eu me pergunto o que você } \\
\text { pensa". } \\
\text { - Use erros como mistérios para resolver, não como crimes a serem punidos, evitando } \\
\text { culpar participantes por suas ações ou usar um tom de voz crítico ou acusatório. }\end{array}$ \\
\hline Referências & $\begin{array}{l}\text { Cheng A, et al. Faculty Development for Simulation Programs Five Issues for the Future } \\
\text { of Debriefing Training. Simul Healthc } 2015 \text { [citado em } 01 \text { nov 2020]; 10(4):217-222. } \\
\text { DOI: 10.1097/SIH.0000000000000090 } \\
\text { Cheng A, Eppich W, Grant V, Sherbino J, Zendejas B, Cook DA. Debriefing for technology } \\
\text { enhanced simulation: a systematic review and meta-analysis. Med Educ. } 2014 \text { [citado } \\
\text { em } 01 \text { nov 2020]; 48:657-666. DOI:10.1111/medu.12432 } \\
\text { Cheng A, Rodgers DL, Jagt EVD, Eppich W, O'Donnell J. Evolution of the Pediatric } \\
\text { Advanced Life Support course: enhanced learning with a new debriefing tool and Web- } \\
\text { based module for Pediatric. Advanced Life Support instructors. Pediatr Crit Care Med } \\
2012 \text { [citado em } 01 \text { nov 2020]; 13(5):589-595. DOI: } 10.1097 / P C C .0 b 013 e 3182417709 \\
\text { Bowe SN, Johnson K, Puscas L. Facilitation and Debriefing in Simulation Education. } \\
\text { Otolaryngol Clin North Am 2017 [citado em } 01 \text { nov 2020]; 50:989-1001. DOI: } \\
\text { 10.1016/j.otc.2017.05.009 }\end{array}$ \\
\hline
\end{tabular}

